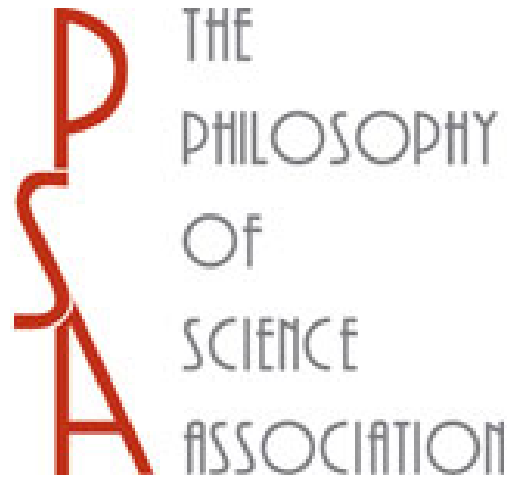

The Limitations of Hierarchical Organization Author(s): Angela Potochnik and Brian McGill

Reviewed work(s):

Source: Philosophy of Science, Vol. 79, No. 1 (January 2012), pp. 120-140

Published by: The University of Chicago Press on behalf of the Philosophy of Science Association

Stable URL: http://www.jstor.org/stable/10.1086/663237

Accessed: 09/02/2012 07:44

Your use of the JSTOR archive indicates your acceptance of the Terms \& Conditions of Use, available at http://www.jstor.org/page/info/about/policies/terms.jsp

JSTOR is a not-for-profit service that helps scholars, researchers, and students discover, use, and build upon a wide range of content in a trusted digital archive. We use information technology and tools to increase productivity and facilitate new forms of scholarship. For more information about JSTOR, please contact support@jstor.org. 


\title{
The Limitations of Hierarchical Organization*
}

\author{
Angela Potochnik and Brian McGill ${ }^{\dagger}$
}

\begin{abstract}
The concept of levels of organization is prominent in science and central to a variety of debates in philosophy of science. Yet many difficulties plague the concept of universal and discrete hierarchical levels, and these undermine implications commonly ascribed to hierarchical organization. We suggest the concept of scale as a promising alternative. Investigating causal processes at different scales allows for a notion of quasi levels that avoids the difficulties inherent in the classic concept of levels. Our primary focus is ecology, but we suggest how the results generalize to other invocations of hierarchy in science and philosophy of science.
\end{abstract}

1. Classic Levels of Organization. The concept of hierarchical organization is commonplace in science and philosophical treatments of science. Though there are different applications of the concept of hierarchy, our primary focus here is the idea that material composition is hierarchical. Subatomic particles compose atoms, which compose molecules; cells compose tissues, which compose organs, which compose organisms; interbreeding organisms compose populations, which compose communities,

*Received January 2011; revised July 2011.

$\dagger$ To contact the authors, please write to: Angela Potochnik, Department of Philosophy, ML 0374, University of Cincinnati, Cincinnati, OH 45221-0374; e-mail: angela .potochnik@uc.edu. Brian McGill, School of Biology and Ecology, Deering Hall 303, University of Maine, Orono, ME 04469; e-mail: mail@brianmcgill.org.

$\$$ This work was conducted as a part of the Function and Evolution Working Group at the National Institute for Mathematical and Biological Synthesis, sponsored by the National Science Foundation, the US Department of Homeland Security, and the US Department of Agriculture through NSF award EF-0832858, with additional support from the University of Tennessee, Knoxville. We received helpful input from the other members of that working group, from two anonymous referees for Philosophy of Science, and from Melinda Fagan. Potochnik also benefited from the feedback of the members of the philosophy of biology lab group at the University of California, Davis, and the audience of a colloquium at Stanford University.

Philosophy of Science, 79 (January 2012) pp. 120-140. 0031-8248/2012/7901-0007\$10.00

Copyright 2012 by the Philosophy of Science Association. All rights reserved. 

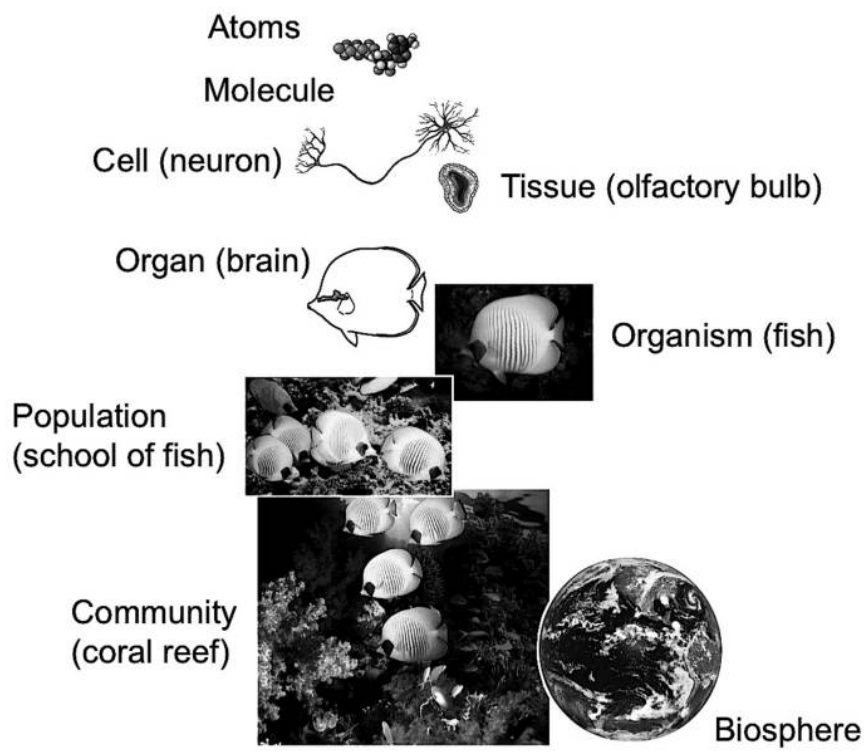

Figure 1. One of the first figures in most ecology textbooks depicts hierarchical levels of organization and asserts their relevance for the study of biology, and especially ecology. Source: Sadava et al. (2008, fig. 1.6).

which compose ecosystems; and so on. The basic idea is that higher-level entities are composed of (and only of) lower-level entities, but the prevalent concept of hierarchical organization involves stronger claims as well. The compositional hierarchy is often taken to involve stratification into discrete and universal levels of organization. It is also often assumed that levels are nested, that is, that an entity at any level is composed of aggregated entities at the next lower level. Intuitively, a population is simply an assemblage of organisms that bear a particular relationship to one another, just as a molecule is simply an assemblage of atoms that bear a (distinct) particular relationship to one another.

This conception of hierarchy is represented in figure 1, which is a reproduction of one of the first figures appearing in a popular undergraduate ecology textbook (Sadava et al. 2008). Indeed, talk of levels is particularly prominent in the field of ecology: nearly all textbooks use the idea that ecological organization is hierarchical as an organizing principle. In addition to Sadava et al.'s book, Ricklefs's The Economy of Nature (2008), possibly the most popular undergraduate ecology textbook, has an in- 
troductory chapter with a description and visual representation of the levels of organization, then has book sections in order of Life and the Physical Environment (basically physiological ecology) followed by Individuals, Populations, Species Interactions, Communities, and finally Ecosystems. Another popular text by Molles (2002) has a nearly identical organization. A third popular ecology text even alludes to hierarchical organization in its title: Ecology: Individuals, Populations, and Communities (Begon, Harper, and Townsend 1986). Editions of these textbooks have been around for decades and have been used to train most practicing ecologists today. (Indeed, the second author learned his introductory ecology from the third book over 25 years ago.) The ecologists writing these textbooks were themselves trained to focus on hierarchical organization. Odum's (1959) ecology textbook describes transitions from "protoplasm to cells, tissues, organs, organ systems, organisms, populations, communities, ecosystems and biosphere" (6).

The concept of hierarchical levels of organization is not merely used to promote basic ecological understanding in students and nonexperts. Hierarchy is a key organizing principle for the field of ecology, particularly since O'Neill et al.'s (1986) influential book conceptualized all of ecology in terms of hierarchy. According to Lidicker (2008, 72), "the nested levels of organization hierarchy has been widely adopted by biologists." The idea of levels of organization is of similar prominence in philosophy of science, and there are striking parallels in how the concept is treated in the two disciplines. For instance, in virtue of the assumption that levels are nested, the ecological hierarchy is sometimes referred to as "the pyramid of life" (Lidicker 2008). This is the apex of the pyramid often used to represent the classic reductionist conception of the whole of science, which ultimately bottoms out at subatomic particles (Oppenheim and Putnam 1958). Yet despite this prominence and analogous treatment of hierarchical organization, there is little agreement in ecology or philosophy about the nature and significance of levels. In this section, we outline a variety of common claims about the nature and significance of hierarchical organization. This prepares the way for section 2 , in which we will develop criticisms of all but the most basic conception of hierarchical composition. Finally, in section 3, we suggest a wholesale replacement for the concept of hierarchy that may accomplish what hierarchy cannot and is less apt to inspire overly strong conclusions.

Feibleman (1954) outlines a classic view of levels of organization that prefigures many later, more nuanced treatments of related issues. His comments provide a convenient starting point for our discussion. Feibleman claims that entities at each successive higher level of organization possess new properties not belonging to their components, for they are particular organizations of their lower-level parts. And yet, any high-level 
object "depends for its continuance" on its lower-level elements (66). A living cell has the property of self-replication, whereas the molecules composing it do not; but there would be no cell without molecules. Feibleman also claims that complexity always increases as levels are ascended while rate of change always decreases. This is related to the last of Feibleman's claims that we will discuss, namely, that organization at a given level is accomplished by a mechanism at the level below, for the purpose it fills at the level above.

Feibleman's comments about distinctive higher-level properties and their dependence on the lower level introduce the issue of the relationship between higher-level and lower-level properties. The idea that higher-level entities possess novel properties is the basis of the idea of emergence. Emergent properties are generally taken to be high-level properties that cannot be predicted, explained, or reduced by lower-level properties, insofar as the organization of components is crucial to the properties' emergence (Kim 1999; Mitchell, forthcoming). The existence and causal relevance of emergent properties are disputed. Less controversial is the idea of mereological supervenience; even proponents of emergentism generally grant that all properties of any (physical) system are determined by the properties of its microphysical components (Horgan 1982; Kim 1999). This metaphysical determination is distinct from causal determination; it is a claim about the metaphysical dependence of higher-level properties on lower-level properties. The idea is that there can be no change in a higher-level property without a corresponding change in one or more lower-level properties. This seems to follow from the compositional relationship of parts to a whole, as long as the parts are all there is to the whole (i.e., barring the possibility of special introductions at higher levels, such as an élan vital or nonphysical soul).

Consider next Feibleman's claims that higher levels of organization are marked by greater complexity and slower rates of change. These ideas have received more attention in ecology than in philosophy. Jagers op Akkerhuis (2008) offers the following brief characterization of the idea that complexity increases with hierarchical organization: "The organization of nature is profoundly hierarchical, because from its beginning, interactions between simple elements have continuously created more complex systems, that themselves served as the basis for still more complex systems" (2). This couples the idea of nested composition with causal production, such that higher-level entities are automatically taken to be more causally complex than their lower-level parts. ${ }^{1}$ O'Neill et al. (1986) rely on similar ideas to justify their idea that hierarchies can be distin-

1. This may in part motivate the widespread idea that biological complexity has increased through evolutionary history (McShea 1991). 
guished by their different rates of change. They claim that higher levels will change more slowly than lower levels (76). Their reasoning seems to be that lower-level interactions amass to result in higher-level behaviors, so the latter must be slower than the former. Though they acknowledge difficulties with the classic view of levels of organization, O'Neill et al. do not clearly distinguish their conception of hierarchical organization from the classic view, and their main examples are also commonly taken to be examples of nested compositional hierarchies. O'Neill et al. further claim that "any attempt to relate a macroscopic property to the detailed behaviors of components several layers lower in the hierarchy is bound to fail due to the successive filtering" (80-81). Detailed arguments for this are developed by O'Neill (1979), and the inability is labeled the "transmutation problem." This is akin to antireductionism about properties, but it instead seems to predict the inability to track causal propagation across levels.

The last of Feibleman's claims that resonates in a useful way with more recent discussions of levels is the idea that the organization of an entity at a given level is accomplished by a mechanism at the level below and that the purpose of that entity's organization is established at the next level above. Some philosophers take mechanisms to be crucial to making sense of the causal significance of levels. Machamer, Darden, and Craver (2000) characterize mechanisms as entities or activities that produce regular changes, and they claim that mechanisms form nested hierarchies. That is, lower-level mechanisms have dedicated roles as parts of higherlevel mechanisms. Craver and Bechtel (2007) argue that mechanisms are key to making sense of all interlevel causal claims. They suggest that causal relationships occur only within a single level, so claims of a causal relationship between entities at different levels actually should be understood as partly a causal claim and partly a constitutive claim. Like O'Neill et al. (1986), these authors articulate a specific basis for hierarchical organization that is distinct from simple composition. However, they suggest that their favored characterization is in fact a version of the traditional compositional hierarchy. ${ }^{2}$

Another type of significance that has been attributed to levels of organization regards scientific explanation. According to explanatory reductionists, higher-level events and regularities should be explained by demonstrating how they arise from lower-level events and regularities

2. According to Craver and Bechtel (2007, 550), "levels of mechanisms are a species of compositional, or part-whole, relations." They cite as evidence some of the very features discussed in this section: that levels of mechanisms are ordered by size, with lower-level entities smaller than higher-level entities, and that levels of mechanisms have slower actions and greater complexity at higher levels. 
(Oppenheim and Putnam 1958; Nagel 1961; Hempel 1966). This thesis is justified with an appeal to the metaphysical dependence of higher-level entities on lower-level entities, that is, mereological supervenience. If all phenomena are determined by entities, causal relationships, and laws at a lower level, then perhaps uncovering lower-level truths - and their relationship to higher levels - is key to explaining higher-level phenomena. Many ecologists look for ecological explanations at lower levels of organization, whether in population dynamics (MacArthur 1968; Wilson and Bossert 1971; May 1976), physiological and behavioral processes (Schoener 1986), or even chemical and physical principles (West and Brown 2005). Arguments against explanatory reductionism also often rely on hierarchical organization, but to opposite effect. They appeal to emergent properties at higher levels, namely, properties that do not reduce to lower-level properties (Mitchell, forthcoming), or to multiply realizable properties, namely, properties that supervene on any one of many distinct lower-level properties (Fodor 1974; Putnam 1975; Garfinkel 1981). More generally, Wimsatt (2007) claims that there is a "level-centered orientation of explanations" (214).

Finally, some explicitly or implicitly consider lower-level theories to be epistemically more secure than higher-level theories. An example is Oppenheim and Putnam's (1958) suggestion that all scientific investigations ultimately may be vindicated by demonstrating their foundation in microphysical law. Such notions of the epistemic value of the lower level are seldom explicitly distinguished from claims of the explanatory value of the lower level. Yet even avowed antireductionists about explanations sometimes assume that physical theory is somehow epistemically privileged over theories in the biological and social sciences. We believe it is a common assumption that if such theories conflict, the higher-level theories should be rejected in favor of the lower-level theories. However, claims to this effect are seldom defended in writing and occur more often in casual conversation. In ecology, population (Wiens 1990) or individual (Schoener 1986) models are clearly privileged over macroecology models. At a seminar when the second author presented his macroecological work, a prominent ecologist responded, "to me, if it is not based in population processes, it is not science."

In this section we have surveyed a range of implications attributed to hierarchical levels of organization. This survey is not exhaustive, but it does capture many of the prominent ideas about the significance of levels. Those ideas can be divided into three categories. First, there are claims about the metaphysical significance of hierarchical levels. These include discussions of mereological supervenience and emergent properties. Second, there are claims about the causal significance of levels. These include ideas put forward about the relative complexity and rates of change of 
different levels as well as the conception of hierarchy in terms of levels of mechanisms. Third, there are claims about the explanatory and evidential significance of levels. These pertain to theories about different levels rather than to entities at different levels of organization. The traditional view of a reductive unity of science falls in this category, but other views embody it as well. For all of these claims about the significance of hierarchical organization, some version of a universal stratification into compositional levels is assumed. ${ }^{3}$ Yet the assumption often remains in the background; as Kim (2002) points out, the conception of levels employed is seldom made explicit.

2. A Vexed Concept. To summarize section 1, the classic formulation of levels of organization in science and philosophy of science is a compositional hierarchy. Each higher-level entity is taken to be an aggregate of entities at the next lower level, so levels are distinguished according to the relationship of parts to a whole. This conception of hierarchical levels is ascribed a variety of implications in various literatures in philosophy and ecology. We have characterized several and grouped them as metaphysical, causal, and evidential/explanatory in nature. In this section, we develop criticisms of all but the most basic conception of the compositional hierarchy. In our view, the many overly ambitious conclusions drawn from the simple fact of part-whole composition - and the persistence of those conclusions - demonstrate that hierarchical stratification is not useful as a general conception of ecology or science.

2.1. Metaphysical Significance. Claims about hierarchical organization often appeal to the ubiquity of part-whole composition. Indeed, the very notion of stratified levels depends on not only the ubiquity, but also the uniformity, of part-whole composition. For strata to emerge, atoms must always compose molecules, populations must always compose communities, and so forth. But the uniformity of composition needed for stratified levels simply does not exist. Guttman (1976), a biologist, forcefully made this point. (Kim [2002] makes the same point, and Wimsatt [2007] argues that uniformity of composition fails at higher levels.) Guttman provides a variety of examples demonstrating that objects at some level $n$ are often not composed exclusively of objects at level $n-1$. For

3. Wimsatt (2007) is an exception. He endorses many of these theses about the significance of levels of organization, but he maintains that decomposability into discrete levels is not universal. Yet, whereas Wimsatt holds the view that "levels of organization are a deep, non-arbitrary, and extremely important feature of the ontological architecture of the natural world" (203), our main thesis is the opposite: that the concept of discrete ontological levels does not succeed and has been used to motivate several problematic claims about the natural world. 
instance, ecosystems are said to be composed of populations or communities, but individual molecules, such as molecules of food waste, are also an important component. Tissues are only partly composed of cells; also crucial are the macromolecules that hold the cells together. Polymer molecules are composed of monomers, but individual ions are also an important ingredient (Guttman 1976).

Care must be taken in articulating the sense in which composition is ubiquitous. It may be that every whole is composed of smaller parts. We do not question that claim here. But it is certainly not the case that every whole is composed of only parts at the next lower level. Nor is it the case that each type of whole is composed of all and only the same types of parts. Consider organisms. Big, lumbering organisms like us are composed of organ systems as well as, for example, cells that often act individually, such as blood cells' role in oxygen transport. At the other extreme, singlecelled organisms are not composed of cells at all; they are composed of cell parts, such as organelles. There is also an array of different compositional configurations between these extremes as well as some entities whose status as organisms is a matter of dispute (Wilson 2008). There is thus reason to call into question the very notion of universal, stratified levels.

Similar difficulties plague the idea that higher-level properties supervene on lower-level properties. We will not dispute the universality of mereological supervenience, that is, the idea that every higher-level property supervenes on lower-level properties. But similar limitations pertain to supervenience as to composition. First of all, each type of higher-level property need not supervene on the same types of lower-level properties. This follows from the well-appreciated phenomenon of multiple realization. Many higher-level properties may supervene on (i.e., be realized by) any of a variety of lower-level properties. For instance, the organism-level property of camouflage is realized by an array of lower-level properties. For many animals, camouflage consists of pigmented cells that disguise through coloration. Other instances of camouflage involve morphological structures of the animal or materials in the environment. The properties of cells, of larger parts of animals, and of nearby materials variously realize organism camouflage by matching backgrounds, concealing shadows, obliterating forms, disguising motion, masquerading as other objects, or creating other perceptual effects (Stevens and Merilaita 2009). Such multiple realization means that any number of types of lower-level properties, of any number of types of lower-level objects, may realize a given higher-level property. Moreover, distinct realizers may very well occupy different levels of organization (Melinda Fagan, personal correspondence).

There is also no guarantee that higher-level properties will supervene 
on a well-defined set of lower-level properties (Mitchell, forthcoming). Even in individual occurrences, higher-level properties may be realized by a complicated combination of lower-level properties that are not naturally grouped as an object of study. One of the authors has termed this complex realization (Potochnik 2010b). To return to the example of camouflage, consider what properties determine an instance of, say, a fish having the property of being camouflaged as a fallen leaf on a stream bed. Relevant properties of the parts of the fish include the properties that determine its overall shape, that it is positioned on its side, and the colors and arrangement of its pigmented cells. Properties of the environment are also relevant: the presence of leaf litter in the stream as well as its heterogeneity, size, and color. Finally, properties of the predators also matter for this to achieve camouflage: predators must be diurnal and aquatic and must hunt by sight (Sazima et al. 2006). The supervenience base of this instance of camouflage - namely, the set of properties that metaphysically determines the presence of the camouflage property - is a complicated set of properties belonging to fish parts, parts of the environment, and parts of predator populations. This set of properties is not naturally ascribed to entities at a single level of organization.

In summary, hierarchical composition may be universal in the sense that every extant whole is composed of proper parts, and mereological supervenience may be universal in the sense that every higher-level property is metaphysically determined by lower-level properties. But stratification into levels is not universal or discrete. Stratification is not discrete in that it is not the case that an object taken to be at some level $n$ is composed of all and only parts at level $n-1$. Stratification is not universal in that it is not the case that all objects taken to be at level $n$ are composed of parts from the same levels $j, \ldots, k$. Similar difficulties plague the metaphysical property determination of the higher level by the lower level, for mereological supervenience is complicated by multiple realization and complex realization. Because of multiple realization, the presence of some high-level property provides little or no information about its determiners, even the level(s) at which its determiners are found; the supervenience base is highly variable from instance to instance. Because of complex realization, the nature of the supervenience base in a given instance is even obscure. There may be a long list of relevant lower-level properties, and they may not all be properties belonging to entities at the same level or even belonging to parts of the object with the higher-level property in question. Even if universal mereological supervenience is a fact, it is not associated with the hierarchical stratification of objects or their properties.

2.2. Explanations and Evidence. Similar difficulties also undermine the supposed explanatory significance of hierarchical stratification. The idea 
of supervenience is at the heart of explanatory reductionism, the view that higher-level events and regularities should be explained by showing how they are metaphysically determined by lower-level events and regularities (Oppenheim and Putnam 1958; Nagel 1961; Hempel 1966). Accordingly, many philosophers have used multiple realization as a basis for arguing against the explanatory priority of the lower level (e.g., Fodor 1974; Putnam 1975; Garfinkel 1981). For multiply realized properties, higher-level regularities exist despite lower-level variations. Antireductionists use this as grounds to dispute the idea that understanding of higher-level phenomena is provided by lower-level information.

The possibility of complex realization - that higher-level properties may be realized by an obscure combination of lower-level properties, as introduced in the section above-further undermines the significance of stratified levels for scientific explanations. The properties that figure into lower-level explanations often fail to be the true supervenience bases of the properties that figure into higher-level explanations. When this is the case, mereological supervenience loses its relevance for the comparison of explanatory strategies. Explanatory reductionists and antireductionists alike err in using supervenience as a way to evaluate the relative value of explanations formulated at different levels. Explanations may be categorized as lower or higher level insofar as they refer to smaller or larger objects-perhaps even parts or wholes. Yet this distinction loses its importance without a basis for comparison (Potochnik 2010b).

The original goal of explanatory reductionism was ultimately epistemic in nature. Oppenheim and Putnam (1958) hoped that all high-level laws could ultimately be derived from - that is, explained by - fully general microphysical laws. This was supposed to vindicate the high-level sciences by showing their basis in microphysics. To Oppenheim and Putnam, the only alternative seemed to be acknowledging nonphysical entities such as the élan vital or nonphysical soul, a repellant proposition for any sort of physicalist (see Potochnik 2011). Though this conception of epistemic vindication via reduction may now be out of favor, we suspect that its ghost lingers in a tendency to credit lower-level theories with greater epistemic security than higher-level theories. This would account for the tendency to assume that higher-level theories should be rejected in favor of lower-level theories when they conflict (discussed in sec. 1). For example, physiological models are often privileged over macroecological models (Schoener 1986), and population genetic models are often privileged over phenotypic optimization models (e.g., Curnow and Ayres 2007). Yet we can think of no basis for privileging lower-level theories in this way. There is no reason to expect that, in general, higher-level theories are less well supported than lower-level theories. For instance, physiological models generally do not incorporate interactions between species, 
such as competition, that are critical to producing the phenomena described in macroecology. Similarly, Potochnik (2010a) shows how population genetic models are not epistemically privileged over purely phenotypic models. For instance, in population genetics, selection coefficients are usually calculated via empirical measure of one fitness component, such as offspring raised to weaning, in lieu of the ecological information that is usually available for phenotypic models.

Not only are lower-level theories often credited with greater epistemic security, they also are seen to have a more general domain of application. Physics is the ultimate example, for it is taken to be the only "fundamental" science. Kim (2002) says, as if stating a universally shared view, that "the domain of physics includes all that there is" (16). But here one must distinguish between physical entities and physical theory. Any physicalist will grant that all objects are composed of only physical stuff. In contrast, it is not obvious - and quite likely false - that theories in physics address all phenomena. It would take the success of a strong reductionist program for any future theories of physics to account for sociological phenomena, for example.

These supposed explanatory and epistemic significances of hierarchical organization arise from a common source, namely, assumptions regarding universal stratified levels, and how lower-level parts and their properties metaphysically determine higher-level objects and their properties. The difficulties with these supposed significances also stem from a single source. Most basically, granting the existence of part-whole composition and mereological supervenience is not sufficient support for the idea that theories and representations are related in these ways. Metaphysical determination is a relation among properties at different levels; this does not straightforwardly dictate the explanatory or epistemic relationship among the theories that have been formulated about phenomena at different levels.

2.3. Levels of Causation. The claims about the significance of levels for relative complexity and rates of change that we described in section 1 rely on the idea that changes at a given level are mediated by lowerlevel processes. Higher-level systems are taken to be more complex since they are created via interactions among simpler lower-level elements. It is also supposed that changes occur more slowly at these higher levels since they are mediated by lower-level changes. Those who defend mechanisms as a way of distinguishing levels articulate a version of this same idea. The conception of nested hierarchies of mechanisms, in which lowerlevel mechanisms perform roles that contribute to some higher-level mechanism, is a particular version of the thesis that changes at a given level are mediated by lower-level processes or, in particular, mechanisms. 
Yet this assumption about the causal significance of levels is flawed, at least when applied to the classic compositional hierarchy. Guttman (1976) argues that, just as an object may have important parts at several different levels of organization, interactions between systems at a given level may be mediated by objects at several different levels. For instance, consider the variety of types of interactions among organisms. One organism may causally influence another via the mediation of pheromones, a type of molecule; may causally produce another via a gamete, a type of cell; or may causally influence another via attack and ingestion, which involves much or all of the organism. ${ }^{4} \mathrm{~A}$ corollary to this point is that causal interactions are not always among systems at the same level of organization - again, at least with regard to the classic compositional hierarchy. Very small objects can causally influence objects taken to be many levels higher: witness the crucial role of waste molecules in an ecosystem. High-level objects can also causally influence objects at much lower levels, what is sometimes termed downward causation (Campbell 1974).

For example, ecology has placed increasing emphasis on migration from a "regional pool" as important in structuring local community dynamics (Hubbell 2001; Magurran and Henderson 2003; McGill 2003; Leibold et al. 2004; Zillio and Condit 2007). In this conceptualization, the regional pool is either an explicit assemblage of many communities known as a metacommunity (Leibold et al. 2004) or an abstract generalization of a much larger area that is structured by different processes (Hubbell 2001; McGill 2003). Similarly, in the study of abundance (the population size of an organism), there is increasingly a move away from the traditional idea that abundance is determined locally through interactions with competitors (MacArthur 1968) and toward the idea that global abundance (abundance of a species across its entire range), shaped by processes such as the evolution of specialists versus generalists, is a crucial determiner (Gaston and Blackburn 2000; Gregory 2000; White et al. 2007). Finally, in molecular and cellular biology, there has been a growing disenchantment with the inability of the reductionist approachthat is, a focus on single, isolated molecules - to explain medically important but holistic disease concepts such as "metabolic syndrome" (linking heart disease, diabetes, obesity, etc.). There is now a trend toward placing individual molecules into a systemic context known as "systems biology" (Kitano 2002).

This undermines the idea that all changes are mediated by causal processes solely at a lower level, thereby removing the basis of the claim

4. Throughout this discussion, we assume that there are high-level causal relationships. 
that complexity always increases as the hierarchy is ascended. And there is indeed reason to doubt that compositional levels are always ordered according to their causal complexity. Strevens (2006), for instance, proposes an account of how simple behavior emerges in high-level systems, even when the parts of the systems interact in complex ways. One of Strevens's primary examples is the relatively simple behavior of whole ecosystems, as seen in the wide applicability of, for example, the LotkaVolterra equations for predator-prey cycles.

There is also no reason to conclude that rates of change always slow as the hierarchy is ascended. Consider the sudden extinction of a large population of organisms or even an entire ecosystem. In contrast, genotypes are exceedingly conservative, and the evolution of a new gene complex can take thousands of generations. The type of change in question is more significant for its rate than is the size of the entities involved.

Abandoning the idea that changes are always mediated by lower-level processes undermines the idea that mechanisms provide a way to distinguish among levels of organization. The parts and wholes of the classic compositional hierarchy do not uniformly constitute nested levels of mechanisms. It is possible to provide an alternative definition of levels in terms of mechanisms. Craver and Bechtel (2007), for instance, explicitly distinguish levels according to the components of mechanisms. But whereas Craver and Bechtel suggest levels of mechanisms as a species of the compositional hierarchy (see n. 2 above), it must instead be emphasized that this conception of mechanism levels does not cohere to the classic conception of hierarchy. (Craver [2007] does more to establish that distinction.) Furthermore, the division of levels of mechanism is variable, depending on what causal processes - namely, what mechanisms - are focal.

3. Replacing Levels with Scale. Let us consider where we have arrived. Though the concept of hierarchical levels of organization crops up in an impressive array of philosophical and scientific contexts, the various significances attributed to it almost entirely fail. The simple fact of partwhole composition is not a sound basis for the assertion of the universal stratification of objects and their properties into discrete levels, or the explanatory or evidential significance of this supposed stratification. Many of the supposed causal significances of hierarchical organization also fail to obtain.

These failures are extensive, but still the concept of hierarchical levels of organization persists or, more accurately, many concepts of hierarchy persist. These include, for example, nested levels of mechanism (Machamer et al. 2000; Craver and Bechtel 2007), hierarchical rates of change (O’Neill et al. 1986), and behavioral composition (Rueger and McGivern 
2010). Different conceptions of hierarchical organization do not obviously align, and these raise the question of whether, or to what extent, they cohere. As Rueger and McGivern put the point, "we might wonder whether one hierarchy or ordering is genuine and the others merely convenient, or indeed whether all hierarchies have no ontological significance and are merely different modes of representing an un-layered reality" (380). We suggest the latter. The search for a universal hierarchical ordering with any broad significance is futile and should be replaced by an approach that explicitly limits its aims to useful quasi-hierarchical representations. ${ }^{5}$

There are significant reasons to abandon the concept of a single, ontologically significant system of hierarchical organization. It has historically been associated with an astounding range of implications, few or none of which are borne out, as demonstrated in section 2 . The very formulation of the concept suggests universality and, thereby, ontological significance, both of which we have called into doubt. We therefore suggest abandoning the terminology, and the concept, of hierarchical levels of organization. We suggest that the concept of scale take its place as an organizing scheme for ecology, and perhaps in science more broadly.

3.1. Ecological Scale. Our suggested replacement for the concept of hierarchy stems from current ecology. By some measures, the most heavily cited paper in ecology today is Levin (1992) on the role of scale in ecology. Levin asserts that "the problem of relating phenomena across scales is the central problem in biology and in all of science" (1961; see also Wiens 1989; Schwartz 2002). Scale is the spatial or temporal extent across which observations span. That is, it is the size of the "ruler" used to measure a system, the choice of which influences the type of observations made. Ecology is sometimes studied at the scale of square meter plots of grass; other ecological studies span entire continents or the globe (a scale of $1,000-10,000$ kilometers to a side, or $10^{12}-10^{14}$ square meters). Temporal scales and the spatial scales often covary - processes occurring at large spatial scales often also occur on long timescales-but there are exceptions. ${ }^{6}$

This recent focus on scale is a response to a trend in ecology during

5. Our argument does not prohibit hierarchical representations that have an explicit basis for ranking and are limited in scope and domain of application. However, any concept constrained in these ways is not well positioned to stand in for classical levels of organization.

6. Note that the term "large scale" can have opposite meanings: to a geographer, a "large" scale actually focuses on a small area. Here we intend the sense commonly used by ecologists, according to which a large scale regards a large area. 
the 1970s and 1980s, when a strong drive for experimentation as the dominant modality had the unintended consequence of research focused only on small spatial and temporal scales, since those were amenable to experimental manipulation. Most experiments in ecology are conducted at the scale of a few square meters at most (Maurer 1999). This focus on a single, relatively small scale remained largely unanalyzed, but if pressed, researchers would justify it by analogy with physics. In physics, experiments on small systems can be informative across many orders of magnitude of scale (though this too can fail; Rueger and McGivern 2010). For example, Galileo's experiments with balls rolling down ramps at a scale of a few meters and Brahe's data on planetary orbits at scales of $10^{12}$ meters both informed and are well described by Newton's three laws of gravity, though they span 11 orders of magnitude of scale.

In the 1990s ecologists began to address larger scales again, largely in response to questions about conservation that concern large scales. Initially, the unspoken assumption was that this would conform to the expectation that experiments done at that small scale would provide an understanding of large-scale phenomena. But there was a growing realization that the processes important at larger scales are often fundamentally different from those observed at lower scales (Wiens 1989; Levin 1992; Schwartz 2002). This set the stage for ecology to become explicitly scale dependent. Individual studies began to specify the scales at which the claims were expected to hold (McGill 2010). As a recent example, McGill suggests that whether or not bird species live in an area is controlled by random chance of dispersing to that area at scales of a few meters, by species interactions at a slightly larger scale, by habitat preferences (e.g., tall trees vs. grass) at scales of tens to hundreds of kilometers, and finally by matches between climate and the species' physiological adaptations at scales approaching those of a continent. Wiens shows that just as causal processes can differ with scale, so can the patterns that are observed. He demonstrates how the abundance of two species of birds covaries negatively at small scales (10 meters), because of competition for nesting sites, but covaries positively at larger scales (10 kilometers), because of their similar habitat preference for coniferous forests. A change of just three orders of magnitude in scale results in $(a)$ a change in the dominant causal influence - nest site competition versus habitat preference-but more importantly in $(b)$ a change in the very patterns observed-positive versus negative covariance. Clearly ecology was not like physics, and scale is completely central to defining the research question.

Scale is sometimes conflated with levels, and it would be convenient if the two were interchangeable. For example, the influential work by ecologists O'Neill et al. (1986) is sensitive to the importance of scale, yet they attempt to assimilate scale to the model of discrete levels of orga- 
nization by claiming that the levels are defined by distinct timescales. This approach is shared by the recent suggestion by Rueger and McGivern (2010) about the role of scale in physics. They say that "when physicists talk about levels, they often do not have in mind a mereological ordering of entities. Instead, what they describe is best understood as a stratification of reality into processes or behaviours at different scales" (382). Rueger and McGivern suggest that scale can be used as the basis for an alternative hierarchy, distinct from the compositional hierarchy. These sources add support for the significance of scale and for its generalizability to other fields. We wish to emphasize only that, unlike these authors, we think it is important to fully distinguish between this alternative schema of scale and the idea of a universal hierarchy of discrete levels.

3.2. Reconstructing Quasi Levels. The simple recognition of differences in scale is a well-positioned alternative to the problematic assumption that there is a universal hierarchy of discrete levels. That different treatments succeed at different scales offers a way to demarcate what one might term "quasi levels." In some respects, quasi levels are similar to classic levels: cells occur on a scale of microns to millimeters, while tissues are typically millimeters to decimeters; organisms (vertebrate and angiosperm, at least) are centimeters to many meters; ecosystems are measured in kilometers; and the biosphere is much larger still. Yet the concept of scale and the demarcation of quasi levels that it allows differ from the classical concept in fundamental ways. Most basically, scale is continuous, whereas levels are discrete. This has important implications for how entities are related to one another. While scale gives a directionality (smaller/ bigger) just as levels do (lower/higher), the concept of scale focuses attention on the distance between entities. The question of whether or not that distance is of importance must be treated as an empirical and interestrelative question.

For example, a squirrel may stand 0.1 meter high while a tree that it lives in stands 10 meters high. Considered in terms of the traditional compositional hierarchy, the squirrel and tree are at the same level: the level of individual organisms. Yet their difference in scale is significant for some investigations. Masting occurs when trees produces all their seeds in large bursts, which happens only in some years. For investigations of the evolution of masting, the salient relationship is between a population of squirrels and an individual tree. One squirrel does not eat enough seeds to drive trees to evolve masting; it takes an entire population. Indeed, the time between masts has evolved to time periods roughly equal to the generation time of a squirrel (about 2 years) so as to have maximal influence on the population dynamics of the squirrel while minimizing influence on the tree's fitness. In contrast, for investigations of populations' 
northward progression in response to global climate change, the differences in size between a squirrel and a tree are insignificant. Their dispersal distances are very similar; indeed there is evidence that the dispersal rate of oak populations is heavily dependent on the dispersal of seeds by squirrels (Corre et al. 1997; Clark 1998).

A few features of this example are illustrative. First, one level from the traditional hierarchy (e.g., individual squirrel and tree) can span several orders of magnitude of scale (here 0.1 vs. 10 meters), just as entities of different compositional levels (e.g., squirrel population and tree) can be of the same scale. Second, the difference in scale between the squirrel and the tree is important in one scenario (masting evolution) but not in the other (dispersal). Whether differences in scale are significant depends on both the research question and the system under investigation. Quasi levels are context dependent: they are explicitly defined relative to a particular domain and set of interests. Differences in scale are not domain and interest relative; the relativity arises in where to locate boundaries in scale. The determination of what range of scales to group together depends on how the causal relationships under investigation behave: what entities or groups of entities interact, at what scale different causal processes become dominant, and so forth. There is therefore no expectation that a successful demarcation of quasi levels has ontological significance or even significance for unrelated phenomena. This is an important departure from classic conceptions of hierarchical levels.

Another key difference between classic levels and quasi levels determined by scale is that the former elevates part-whole relationships to prominence, while scale is fully independent of part-whole relationships. The focus on composition is replaced by a focus on causal processes or interactions. Put in terms of the previous example, one squirrel cannot digest enough acorns to influence a large oak; in this case, significant causal interaction requires a population of squirrels. Indeed, types of causal interaction sometimes can be correlated with scale. Organisms tend to compete with organisms that are similar in size, to eat organisms approximately an order of magnitude smaller, and to be infected by organisms about two or three orders of magnitude smaller (Peters 1983). ${ }^{7}$

This demonstrates that the analysis of causal relationships is central to demarcating quasi levels. Yet the significance of causality is markedly

7. Our suggestion that types of causal interaction are sometimes correlated with scale is evocative of what Wimsatt (2007) terms "causal thickets" and the partial perspectives they engender. In Wimsatt's view, causal thickets are the alternative to well-defined levels, for though he takes levels of organization to be an "extremely important feature of the ontological architecture of our natural world" (203), he does not posit the universality of levels (see n. 3 above). 
TABle 1. Comparison of Classic Levels and Quasi Levels DEMARCATED FROM SCALE

\begin{tabular}{lll}
\hline & \multicolumn{1}{c}{ Classic Levels } & \multicolumn{1}{c}{ Quasi Levels } \\
\hline Measure & Discrete & Continuous \\
Boundaries & Universal & Domain relative \\
Based on & Composition & Causal processes \\
Causal significance & Causation only within a level & Causation among all scales \\
Temporal scale & Slower change at higher levels & Independent of spatial scale \\
\hline
\end{tabular}

different from the causal significance attributed to classic levels of organization. First, we recommend using analysis of causal relationships to demarcate quasi levels, whereas with classical levels, the direction is reversed: causation is supposed to occur within a level, so where to expect causal relationships depends on the structure of the universal hierarchy. Second, one may find causal interactions among entities or groups at any range of scales, though there may of course be patterns in types of interactions at different scales. Notice especially that small-scale phenomena can be influenced by, and thus illuminated by, large-scale events. For the example above, whether oaks can evolve a local adaptive response to squirrels eating their acorns depends in part on gene flow from oaks in many other regions, for this may swamp local selective pressures. In contrast, the classic hierarchy has been used to motivate the idea that causal interactions occur only at a single level and are mediated by the next lower level. Downward causation especially has been the target of much criticism. Third, the concept of scale can structure investigations according to both spatial scale and temporal scale, but correlation between these two scales cannot be assumed. This is in contrast to the expectation that classic compositional levels are related to rate of change. These differences between classic levels and quasi levels are depicted in table 1 .

We hope to have demonstrated that the concept of scale, along with the derivative concept of quasi levels, offers a superior conceptual framework over traditional hierarchical levels for conducting science, and especially ecology. We have not established that quasi levels in scale are the only possible conceptual replacement for traditional levels, but we have suggested ways in which these concepts do work similar to that of classical levels while avoiding the latter's pitfalls. Quasi levels, demarcated according to the central concept of scale, provide a conception of organization that is overtly interest relative and problem dependent. Part-whole composition may be universal, but it does not proceed in lockstep, and it is not the sole structuring relationship among phenomena-or the most significant. Replacing classical levels of organization with considerations of scale brings the analysis of causality to the fore and allows causal significance to govern the delineation of levels. 


\section{REFERENCES}

Begon, Michael, John L. Harper, and Colin R. Townsend. 1986. Ecology: Individuals, Populations, and Communities. Sunderland, MA: Sinauer.

Campbell, Donald T. 1974. "'Downward Causation' in Hierarchically Organised Biological Systems." In Studies in the Philosophy of Biology, ed. F. Ayala and T. Dobzhansky, 179-86. Berkeley: University of California Press.

$\rightarrow$ Clark, James S. 1998. "Why Trees Migrate So Fast: Confronting Theory with Dispersal Biology and the Paleorecord." American Naturalist 152:204-24.

Corre, Valerie Le, Nathalie Machon, Remy J. Petit, and Antoine Kremer. 1997. "Colonization with Long-Distance Seed Dispersal and Genetic Structure of Maternally Inherited Genes in Forest Trees: A Simulation Study." Genetical Research 69:117-25.

Craver, Carl F. 2007. Explaining the Brain: Mechanisms and the Mosaic Unity of Neuroscience. Oxford: Oxford University Press.

Craver, Carl F., and William Bechtel. 2007. "Top-Down Causation without Top-Down Causes." Biology and Philosophy 22:547-63.

Curnow, R. N., and K. L. Ayres. 2007. "Population Genetic Models Can Be Used to Study the Evolution of the Interacting Behaviors of Parents and Their Progeny." Theoretical Population Biology 72:67-76.

Feibleman, James K. 1954. "Theory of Integrative Levels." British Journal for the Philosophy of Science 5 (17): 59-66.

Fodor, Jerry. 1974. "Special Sciences: The Disunity of Science as a Working Hypothesis." Synthese 28:97-115.

Garfinkel, Alan. 1981. Forms of Explanation: Rethinking the Questions in Social Theory. New Haven, CT: Yale University Press.

Gaston, Kevin J., and Tim M. Blackburn. 2000. Pattern and Process in Macroecology. Oxford: Blackwell Scientific.

$\rightarrow$ Gregory, Richard D. 2000. "Abundance Patterns of European Breeding Birds." Ecography 23:201-8.

Guttman, Burton S. 1976. "Is 'Levels of Organization' a Useful Biological Concept?" BioScience 26 (2): 112-13.

Hempel, Carl. 1966. Philosophy of Natural Science. Englewood Cliffs, NJ: Prentice-Hall.

Horgan, Terence E. 1982. "Supervenience and Microphysics." Pacific Philosophical Quarterly 63:29-43.

Hubbell, Stephen P. 2001. A Unified Theory of Biodiversity and Biogeography. Princeton, NJ: Princeton University Press.

Jagers op Akkerhuis, Gerard A. J. M. 2008. "Analysing Hierarchy in the Organization of Biological and Physical Systems." Biological Reviews 83:1-12.

$\rightarrow$ Kim, Jaegwon. 1999. "Making Sense of Emergence." Philosophical Studies 95 (1-2): 3-36.

$\rightarrow$. 2002. "The Layered Model: Metaphysical Considerations." Philosophical Explorations 5 (1): 2-20.

$\rightarrow$ Kitano, Hiroaki. 2002. "Systems Biology: A Brief Overview." Science 295:1662.

$\rightarrow$ Leibold, M. A., et al. 2004. "The Metacommunity Concept: A Framework for Multi-Scale Community Ecology." Ecological Letters 7:601-13.

Levin, Simon A. 1992. "The Problem of Pattern and Scale in Ecology." Ecology 73 (6): 1943-67.

Lidicker, William Z., Jr. 2008. "Levels of Organization in Biology: On the Nature and Nomenclature of Ecology's Fourth Level." Biological Reviews 83:71-78.

MacArthur, Robert H. 1968. "The Theory of the Niche." In Population Biology and Evolution, ed. Richard C. Lewontin, 159-76. Syracuse, NY: Syracuse University Press.

Machamer, Peter, Lindley Darden, and Carl F. Craver. 2000. "Thinking about Mechanisms." Philosophy of Science 67 (1): 1-25.

Magurran, Anne E., and Peter A. Henderson. 2003. "Explaining the Excess of Rare Species in Natural Species Abundance Distributions." Nature 422:714-16.

Maurer, Brian A. 1999. Untangling Ecological Complexity. Chicago: University of Chicago Press. 
May, R. M. 1976. "Models for Two Interacting Populations.” In Theoretical Ecology: Principles and Applications, ed. R. M. May, 49-70. Philadelphia: Saunders.

McGill, Brian J. 2003. "Does Mother Nature Really Prefer Rare Species or Are Log-LeftSkewed SADs a Sampling Artefact?" Ecological Letters 6:766-73.

$\rightarrow-$. 2010. "Ecology: Matters of Scale." Science 328:575.

$\rightarrow$ McShea, Daniel W. 1991. "Complexity and Evolution: What Everybody Knows." Biology and Philosophy 6:303-24.

Mitchell, Sandra D. Forthcoming. "Emergence: Logical, Functional and Dynamical.” Synthese.

Molles, Manuel C. 2002. Ecology: Concepts and Applications. Boston: McGraw-Hill.

Nagel, Ernest. 1961. The Structure of Science. London: Routledge \& Kegan Paul.

Odum, Eugene P. 1959. Fundamentals of Ecology. 2nd ed. Philadelphia: Saunders.

O’Neill, Robert V. 1979. "Transmutations across Hierarchical Levels." In Systems Analysis of Ecosystems, ed. G. S. Innes and Robert V. O'Neill, 59-78. Fairland, MD: International Co-operative.

O’Neill, Robert V., D. L. DeAngelis, J. B. Waide, and T. F. H. Allen. 1986. A Hierarchical Concept of Ecosystems. Princeton, NJ: Princeton University Press.

Oppenheim, Paul, and Hilary Putnam. 1958. "Unity of Science as a Working Hypothesis." In Minnesota Studies in the Philosophy of Science, vol. 2, ed. Herbert Feigl, Michael Scriven, and Grover Maxwell, 3-36. Minneapolis: University of Minnesota Press.

Peters, Robert H. 1983. The Ecological Implications of Body Size. Cambridge: Cambridge University Press.

Potochnik, Angela. 2010a. "Explanatory Independence and Epistemic Interdependence: A Case Study of the Optimality Approach." British Journal for the Philosophy of Science 61 (1): $213-33$.

$\rightarrow-2010 b$. "Levels of Explanation Reconceived." Philosophy of Science 77 (1): 59-72. 19.

Putnam, Hilary. 1975. "Philosophy and Our Mental Life." In Philosophical Papers, vol. 2, Mind, Language and Reality, 291-303. Cambridge: Cambridge University Press.

Ricklefs, Robert E. 2008. The Economy of Nature. 6th ed. New York: Freeman.

$\rightarrow$ Rueger, Alexander, and Patrick McGivern. 2010. "Hierarchies and Levels of Reality." Synthese 176:379-97.

Sadava, David, H. Craig Heller, Gordon H. Orians, William K. Purves, and David Hillis. 2008. Life: The Science of Biology. 8th ed. Sunderland, MA: Sinauer.

Sazima, Ivan, Lucelia Nobre Carvalho, Fernando Pereira Mendonca, and Jansen Zuanon. 2006. "Fallen Leaves on the Water-Bed: Diurnal Camouflage of Three Night Active Fish Species in an Amazonian Streamlet." Neotropical Ichthyology 4 (1): 119-22.

Schoener, Thomas W. 1986. "Mechanistic Approaches to Community Ecology: A New Reductionism." American Zoologist 26:81-106.

Schwartz, James. 2002. "Population Genetics and Sociobiology." Perspectives in Biology and Medicine 45 (2): $224-40$.

Stevens, Martin, and Sami Merilaita. 2009. "Animal Camouflage: Current Issues and New Perspectives." Philosophical Transactions of the Royal Society B 364:423-27.

Strevens, Michael. 2006. Bigger than Chaos: Understanding Complexity through Probability. Cambridge, MA: Harvard University Press.

$\rightarrow$ West, Geoffrey B., and James H. Brown. 2005. "The Origin of Allometric Scaling Laws in Biology from Genomes to Ecosystems: Towards a Quantitative Unifying Theory of Biological Structure and Organization.” Journal of Experimental Biology 208 (9): 157592.

White, Ethan P., S. K. Morgan Ernest, Andrew J. Kerkhoff, and Brian J. Enquist. 2007. "Relationships between Body Size and Abundance in Ecology." Trends in Ecology and Evolution 22:323-30.

Wiens, John A. 1989. "Spatial Scaling in Ecology.” Functional Ecology 3:385-97. 1990. "Ecology 2000: An Essay on Future Directions in Ecology." Revista Chilena de Historia Natural 65:309-15. 
Wilson, Edward O., and William H. Bossert. 1971. A Primer of Population Biology. Sunderland, MA: Sinauer.

Wilson, Robert A. 2008. "The Biological Notion of Individual." In The Stanford Encyclopedia of Philosophy, ed. Edward N. Zalta. Stanford, CA: Stanford University. http:// plato.stanford.edu/archives/fall2008/entries/biology-individual.

Wimsatt, William C. 2007. Re-engineering Philosophy for Limited Beings. Cambridge, MA: Harvard University Press.

$\rightarrow$ Zillio, Tommaso, and Richard Condit. 2007. "The Impact of Neutrality, Niche Differentiation and Species Input on Diversity and Abundance Distributions." Oikos 116:93140. 